\title{
Empatía: Medidas Cognitivas y Psicofisiológicas en Población Infantil
}

\section{Empathy: Cognitive And Psychophysiological Measures In The Child Population}

Duván Fernando Gómez-Morales, ${ }^{2}$ Jasmin Bonilla-Santos, ${ }^{1}$ Daniela Gutiérrez-Sterling, ${ }^{2}$ Alfredis González-Hernández, Gisella Bonilla-Santos²

\begin{abstract}
Resumen
La empatía ha consolidado una importante trayectoria investigativa desde el siglo pasado en las neurociencias cognitivas. Desde su definición como concepto, la construcción de instrumentos de evaluación para diferentes grupos de edad y la búsqueda de dominios y vertientes que aborden este componente no ha cesado. Esta revisión se dirige a recopilar los hallazgos representativos en la historia de la conceptualización del término y el desarrollo de la medición en empatía en las poblaciones, haciendo especial énfasis en los trabajos realizados con población infantil con herramientas de autoinforme y medidas psicofisiológicas. La revisión arrojó que existe un horizonte investigativo prometedor de la mano de técnicas psicofisiológicas como el EEG para la evaluación del constructo. Se discute la necesidad de profundizar en la búsqueda de patrones en la ontogenia de la empatía en la niñez, así como en el diseño de nuevas formas de medición a través de autoinforme para la práctica investigativa en países de habla hispana que involucre las dinámicas sociales y demográficas de estas poblaciones.
\end{abstract}

Palabras clave: empatía, cognición, neuropsicología

\section{Abstract}

Empathy has an important research trajectory since the last century in cognitive neurosciences. From the definition as a concept, the construction of evaluation instruments for different ages and the search for domains and aspects that address this component are still in force. This review aims to collect representative findings in the history of the conceptualization of the term and the development of measurement in empathy in populations, with special emphasis on the work carried out with the child population with self-report tools and psychophysiological measures. The review showed that there is a promising research horizon with psychophysiological techniques such as the EEG for the evaluation of the construct. The need to deepen the search for patterns in the ontogeny of empathy in childhood is discussed, as well as the design of new forms of measurement through selfreport for research practice in Spanish-speaking countries that involve social dynamics and demographics of these populations.

Keywords: empathy, cognition, neuropsychology

Rev. Ecuat. Neurol. Vol. 30, N 1, 2021

\section{Introducción}

El estudio de la empatía durante los últimos años ha consolidado nuevas perspectivas investigativas en el área de las neurociencias cognitivas. El primer antecedente del concepto de empatía se origina a finales del siglo XIX en Alemania, cuando el historiador del arte Robert Vischer en el año 1973 utiliza la palabra alemana Einfuhlung para discutir sobre la psicología de la estética y la percepción de la forma.' Posteriormente, en el año 1909, Titchener traduce para la psicología experimental estadounidense la palabra al inglés "empathy," y es a partir de esta fecha que diversos autores como Lipps, Diamon, Mehrabian, Kholer, Hoffman, entre otros empiezan a desarrollar estudios para explicar conceptualmente este proceso; así como realizar investigaciones con el objetivo de encontrar la relación con la conducta prosocial, ${ }^{2-5}$ a explorar las características empáticas del personal médico con los pacientes ${ }^{6-8}$ y a estudiar la empatía en pacientes que tienen rasgos psicopáticos. ${ }^{90}$ Como resultado de estos antecedentes, el uso del término en las áreas de las neurociencias y en la psicología ha consolidado una trayectoria importante generando variedad en los conceptos y una discusión que se mantiene vigente. ${ }^{11-13}$ Por ello, el objetivo del presente artículo es proporcionar un análisis de la diversificación en el concepto y medición de la empatía, específicamente en población infantil, y presentar las medidas de evaluación de empatía con los métodos psicofisiológicos.
'Universidad Cooperativa de Colombia, Campus Neiva, Departamento de Psicología-Huila, Colombia.

${ }^{2}$ Universidad Surcolombiana, Facultad de Ciencias Humanas y Sociales. Neiva-Huila, Colombia.
Correspondencia:

Jasmin Bonilla-Santos, Universidad Cooperativa de Colombia, Calle 11 No. 1g-31Neiva-Huila, Colombia. Teléfono: 057- 8725900.

E-mail: jasmin.bonillas@campusucc.edu.co 
Los métodos utilizados para evaluar la empatía están determinados por una base conceptual consolidada, iniciando con investigaciones principalmente enfocadas a la población adulta. Aunque en la literatura se registran antecedentes a finales del siglo XIX, es a partir del año 1948 que Rosalind Dymond propuso una descripción conceptual de la empatía, entendida como la capacidad de tomar el rol del otro en las relaciones interpersonales..$^{14}$ Más adelante en 1949, la autora añadió que la empatía era la transposición imaginativa de uno mismo en el pensamiento, sentimiento y actuación del otro; la anterior definición tuvo una tendencia a ser similar con los términos de conciencia social, simpatía o percepción del otro.

Las conclusiones procedentes de aquella época conllevaron a que Hogan considerara que el término hasta el momento era impreciso, debido a que existía una brecha entre la teoría y la medición, además que la empatía aludía al proceso de construcción propia del estado mental de otra persona ${ }^{15}$ Con el objetivo de hallar una definición actualizada y precisa para la época, solicitó a cuatro psicólogos investigadores y a tres estudiantes de psicología de la Universidad de California que seleccionaran los ítems del California Q-Sort Test de Block ${ }^{16}$ relacionados con una persona altamente empática. Como resultado, Hogan conceptualizó que una persona empática se caracterizaría por ser socialmente perceptiva de un amplio repertorio de claves interpersonales, sería consciente de la impresión que causa a los demás, poseería habilidades en técnicas sociales de juego imaginativo, fingimiento y humor, sería consciente de los motivos y el comportamiento propio, y evaluaría la motivación de otros en situaciones de interpretación.

Para el año 1969, surgen los aportes teóricos de Stotland que consideró que pese a que la empatía se podía comprender desde lo cognitivo, la forma en que el sujeto percibe y piensa en el otro está mediada por la relación social $1{ }^{17}$ Considerando lo anterior, Mehrabian y Episten en 1972 le otorgan a la empatía una definición que se relaciona con el componente emocional como una respuesta emocional vicaria a las experiencias emocionales percibidas de los demás..$^{18}$ Además de la propuesta conceptual innovadora, los autores hallaron que cuando se experimenta un alto nivel de empatía emocional se reduce la probabilidad de tener un comportamiento agresivo, y que percibir de forma inmediata las señales de dolor de la víctima, aumenta la posibilidad de que un sujeto participe en el comportamiento de ayuda.

Posteriormente, Mark Davis en los años 80 desarrolló una medida de empatía multidimensional con el objetivo de incluir el componente cognitivo y emocional a través de cuatro aspectos o escalas. ${ }^{19}$ El componente cognitivo se conceptualizó con la escala de toma de perspectiva que permite medir la tendencia de adoptar espontáneamente el punto de vista psicológico de los demás, incorporado con la escala de fantasía, que mide la tendencia a transponerse imaginativamente en los sentimientos y acciones de personajes ficticios. Para el componente emocional, diseñó las escalas de preocupación empática o preocupación por los demás y la escala de angustia emocional, elaboradas para medir los sentimientos orientados a la ansiedad personal y malestar en situaciones interpersonales.

Continuando en el enfoque multidimensional, en el año 2004 Baron-Cohen y Wheelwright ${ }^{20}$ argumentaron que no era posible hablar de empatía desde un solo enfoque dado que estos coexisten, de manera que la empatía se entiende como la capacidad de atribuir estados mentales a otra persona/animal que a su vez conlleva a que exista una respuesta afectiva apropiada en el observador al estado mental de otro individuo.

Por otro lado, Decety y Jackson ${ }^{21}$ proponen un modelo de explicación donde la empatía está compuesta por tres componentes funcionales que interactúan dinámicamente para producir la experiencia empática en humanos. El primer componente es el intercambio afectivo entre el yo y el otro, basado en el acoplamiento percepción-acción que conduce a representaciones compartidas; el segundo componente es la conciencia del otro, y, por último, la capacidad de flexibilidad mental para adoptar la perspectiva subjetiva del otro que complementa los procesos regulatorios. Estos tres procesos están entrelazados entre sí para producir la experiencia subjetiva de la empatía.

De esta manera la discusión de los investigadores se dirigió en establecer si la empatía era cognitiva, afectiva o multidimensional. Sumado a lo anterior, se construyó, se validó y se aplicó pruebas para la evaluación de este constructo enfocadas en el componente cognitivo con aportes de autores como Dymond, Ker \& Speroff, y Hogan; ;4,15,22 en el componente afectivo Mehrabian \& Epstein, Bryant, Caruso \& Mayer, Joliffe y Farrington; ${ }^{18,23-25}$ finalmente con un enfoque multidimensional Davis, y Baron-Cohen \& Wheelwright. ${ }^{19,20}$

\section{Métodos de evaluación de autoinforme de empatía en población infantil}

En la literatura, el Índice de Reactividad Interpersonal (IRI) de Davis $^{26}$ es el instrumento de autoinforme más utilizado y traducido por diversos autores para medir la empatía en población adulta ${ }^{27-34}$ Aunque la creación de los ítems en su versión original en inglés se realizó para población adulta, la prueba es utilizada para múltiples investigaciones en población infantil $l^{35-41}$ La prueba original específicamente evaluó 475 estudiantes universitarios de psicología de Estados Unidos y contiene 28 ítems que se distribuyen en cuatro dimensiones que abarcan el dominio cognitivo y afectivo de la empatía. El test es ampliamente utilizado por sus propiedades psicométricas, toda vez que reporta una confiabilidad interna que varía entre 0,71 a 0,77 y la confiabilidad mediante test-re test de 0,62 a 0,71 . 
Además esta prueba ha sido adaptada para población adulta hispanohablante en una muestra de 692 personas y en una muestra de estudiantes universitarios conformada por 2512 individuos en España; ${ }^{42}$ a partir de estos estudios de validación en idioma español se han realizado análisis de validación en otras poblaciones, como la población infantil representada en una muestra de 1.285 niños de 9 a 12 años en Argentina ${ }^{43}$ Este estudio concluyó que la versión argentina tuvo que incluir diferencias a la versión original y a la validación hispanohablante que probablemente se debían a las formas culturales de interpretación de los ítems. Pese a que existe una versión en castellano y dirigida a población infantil, no todos los reportes utilizan la versión argentina del IRI.

A su vez Mehrabian y Epstein proponen el Cuestionario de Medida de Tendencia Empática (QMME) ${ }^{18}$ y exploraron la validez de la medida en experimentos que involucraban agresión y comportamientos de ayuda. Esta escala obtuvo un coeficiente de correlación Split-half de 0.84 en una población de 202 estudiantes de una clase de psicología de la Universidad de California. Con este test se realizaron estudios posteriores en población de estudiantes de secundaria con el objetivo de evaluar el comportamiento de ayuda y la conducta moral ${ }^{44}$ así como evaluar la relación entre empatía y el desarrollo moral en adolescentes ${ }^{45}$ Conjuntamente, esta escala ha sido utilizada en la evaluación de déficits en la empatía en niños con distintas condiciones relacionadas con la violencia. ${ }^{46}$

También existen instrumentos que han diseñado sus ítems orientados a población infantil y adolescente. En el año 1982 Brenda Bryant basada en los planteamientos teóricos y metodológicos de Mehrabian y Epstein propone una escala de empatía de auto reporte denominado Índice de Empatía para Niños y Adolescentes (IECA) con un método útil para evaluar niños y adolescentes. La primera muestra de la prueba se trabajó con 258 participantes que cursaban primer, cuarto y séptimo grado; la segunda muestra fue de 72 integrantes de primer grado y 94 de séptimo grado de escuelas del área urbana de una ciudad de Estados Unidos. Bryant tomó 17 ítems de los 33 de la prueba de Mehrabian, y los redactó para ser apropiados para el uso con niños. Por ejemplo, el ítem "a menudo encuentro molestas las demostraciones públicas de afecto" se cambió por "las personas que se besan y abrazan en público son tontas." Así mismo el ítem "me entristece ver un extraño solitario en un grupo" se modificó por "me entristece ver a una niña (niño) que no puede encontrar a nadie con quien jugar." La prueba de 22 ítems de lápiz y papel fue aplicada de manera individual para los niños de primer grado, y de forma grupal para los adolescentes de cuarto y séptimo grado. Los coeficientes de estabilidad mediante test- retest, indicaron un grado adecuado para los estudiantes de primero de 0,74 , cuarto grado 0,81 y de séptimo grado de 0,83 . Según los valores anteriores la prueba cumple con los requisitos mínimos de validez de constructo y ha proporcionado la posibilidad de comprender los antecedentes y condiciones del desarrollo de la empatía desde el componente emocional, aunque se aparta componente cognitivo de la empatía. El IECA ha sido ampliamente utilizado en investigaciones con población infantil y adolescentes en trastornos de conducta, ${ }^{47}$ inteligencia emocional, ${ }^{48}$ y comportamiento prosocial. ${ }^{49}$

Continuando por esta línea de empatía emocional y de trabajo con adolescentes, en el año 1998, Caruso y Mayer crearon una escala que permitiera medir varios componentes de la empatía emocional basada en los postulados teóricos de Mehrabian y Epstein, sin adaptar los ítems del QMME, como si lo realizó Bryant para el IECA. Los autores estaban interesados en utilizar la escala para un programa de investigación sobre inteligencia emocional por lo que su objetivo no era fundamentar el test en el enfoque multidimensional. Se centraron en 6 escalas que hacen parte de la empatía emocional: la escala de sufrimiento, contagio emocional, respuesta al llanto, compartir positivo, sentir por los demás y atención emocional. El puntaje confiabilidad de consistencia mediante Alfa de Cronbach de los 33 ítems fue de 0.88 en una muestra de 290 adolescentes con edades comprendidas entre los 11 a 17 años. Los investigadores sugieren utilizar la escala si la finalidad es evaluar el componente emocional de la empatía, de lo contrario recomiendan utilizar una escala de empatía general.

Contrario a lo anterior, en Inglaterra el año 2006 Jollife y Farrigton proponen una escala para adolescentes que cuente con un enfoque multidimensional, que denominaron Escala de Empatía Básica (BES) inicialmente con 40 ítems aplicada en 363 adolescentes de 15 años. A través de un análisis factorial exploratorio de componentes principales y análisis de rotación varimax reducen la escala a 20 ítems, que un año después se administra de nuevo a 357 adolescentes. La propuesta conceptual del test utiliza la definición de empatía de Cohen y Strayer "como la comprensión y el intercambio en el estado o contexto emocional de otra persona" (p. 523) abarcando la congruencia afectiva y la comprensión de emociones. La prueba también realizó un análisis de validez divergente, que halló una validez en la dirección y magnitud esperada evidenciado en las relaciones observadas con otras pruebas existentes, como la prueba de simpatía, toma de perspectiva, alexitimia, amabilidad, conciencia, apertura, supervisión parental y estado socioeconómico y una validez divergente como lo demuestra la no relación con la respuesta socialmente deseable.

Luego, para investigar la empatía en niños en edad preescolar se creó el Test de Southampton de Empatía para Preescolares (por sus siglas en inglés, STEP). La prueba fue aplicada a 39 niños entre 40 y 53 meses de edad. Como metodología la prueba utilizó 8 viñetas de videos que incluían 4 emociones como enojo, felicidad, 
temor y tristeza que muestra niños en escenarios emocionales. Este método se orientó en evaluar la capacidad del niño para comprender y compartir la experiencia emocional del protagonista de la historia a través de la expresión facial, las situaciones, las señales verbales y el deseo del protagonista. La prueba STEP tuvo una validez concurrente moderada con una escala de empatía calificada por los padres, se realizó análisis de validez de constructo a través de una escala de comportamiento prosocial calificada por los maestros de los participantes y tuvo una buena fiabilidad interna ${ }^{50}$ La Tabla 1 detalla los principales instrumentos de evaluación.

\section{Medidas psicofisiológicas de evaluación de empatía en población infantil}

Los investigadores han explorado las bases fisiológicas de los procesos empáticos que se llevan a cabo en el cerebro utilizando diversas técnicas. Mediante imagen funcional, regiones cerebrales como la ínsula bilateral anterior, el giro anterior del cíngulo, el tronco encefálico, parte del tálamo y el cerebelo ${ }^{51-59}$ han sido vinculadas a los criterios de respuesta de la empatía (Figura1). Adicionalmente, los mecanismos neuronales de la acción de empatizar asociado a la imitación ligada al sistema de neuronas espejo (MNS en sus siglas en inglés) ${ }^{60}$ se han ubicado en el giro

Tabla 1. Instrumentos de evaluación en empatía

\begin{tabular}{|c|c|c|c|c|c|}
\hline & Nombre del instrumento: & País: & Definición de empatia: & Dominio: & Población: \\
\hline 1 & $\begin{array}{l}\text { Rating test of insight and empathy } \\
\text { Dymond (1949) }\end{array}$ & $\begin{array}{l}\text { Estados } \\
\text { Unidos }\end{array}$ & $\begin{array}{l}\text { "Transposición imaginativa de uno mismo en el } \\
\text { pensamiento, sentimiento y actuación del otro". }\end{array}$ & Afectivo & Adultos \\
\hline 2 & $\begin{array}{c}\text { Empathy Test } \\
\text { Kerr y Speroff (1954) }\end{array}$ & $\begin{array}{l}\text { Estados } \\
\text { Unidos }\end{array}$ & $\begin{array}{l}\text { "Capacidad de ponerse en la posición de la otra } \\
\text { persona, establecer una buena relación y } \\
\text { anticipar sus reacciones, sentimientos y } \\
\text { comportamientos". }\end{array}$ & Cognitivo & $\begin{array}{l}\text { Estudiantes } \\
\text { universitarios }\end{array}$ \\
\hline 3 & $\begin{array}{l}\text { Empathy Scale } \\
\text { Hogan (1969) }\end{array}$ & $\begin{array}{l}\text { Estados } \\
\text { Unidos }\end{array}$ & $\begin{array}{l}\text { "La aprehensión intelectual o imaginativa de la } \\
\text { condición o estado mental de otra persona sin } \\
\text { experimentar realmente los sentimientos de esa } \\
\text { persona". }\end{array}$ & Afectivo & $\begin{array}{l}\text { Adultos y } \\
\text { estudiantes de } \\
\text { secundaria }\end{array}$ \\
\hline 4 & $\begin{array}{l}\text { Measure of Emotional Empathy } \\
\text { Mehrabian y Epstein (1972) }\end{array}$ & $\begin{array}{l}\text { Estados } \\
\text { Unidos }\end{array}$ & $\begin{array}{l}\text { "Respuesta emocional vicaria a las experiencias } \\
\text { emocionales percibidas de los demás". }\end{array}$ & Afectivo & $\begin{array}{l}\text { Estudiantes } \\
\text { Universitarios }\end{array}$ \\
\hline 5 & $\begin{array}{c}\text { Interpersonal Reactivity Index } \\
\text { Davis (1980) }\end{array}$ & $\begin{array}{l}\text { Estados } \\
\text { Unidos }\end{array}$ & $\begin{array}{l}\text { "Se refiere a las reacciones de un individuo al } \\
\text { observar experiencias de otro" }\end{array}$ & $\begin{array}{l}\text { Cognitivo- } \\
\text { Afectivo }\end{array}$ & Adultos \\
\hline 6 & $\begin{array}{l}\text { An Index of Empathy for Children } \\
\text { and Adolescents Bryants (1982) }\end{array}$ & $\begin{array}{l}\text { Estados } \\
\text { Unidos }\end{array}$ & $\begin{array}{l}\text { "Respuesta emocional vicaria a las experiencias } \\
\text { emocionales percibidas de los demás". }\end{array}$ & Afectivo & $\begin{array}{c}\text { Niños y } \\
\text { adolescentes }\end{array}$ \\
\hline 7 & $\begin{array}{l}\text { Measure of Emotional Empathy for } \\
\text { Adolescent and Adults } \\
\text { Caruso y Mayer (1998) }\end{array}$ & $\begin{array}{l}\text { Estados } \\
\text { Unidos }\end{array}$ & "Empatía como respuesta emocional". & $\begin{array}{l}\text { Cognitivo- } \\
\text { Afectivo }\end{array}$ & $\begin{array}{l}\text { Adolescentes y } \\
\text { adultos }\end{array}$ \\
\hline 8 & $\begin{array}{c}\text { Escala de Empatía e Identificación } \\
\text { con los personajes } \\
\text { Igartua y Páez (1998) }\end{array}$ & España & $\begin{array}{l}\text { "Empatía afectiva, capacidad de volverse el/la } \\
\text { protagonista, desear ser como los/as } \\
\text { protagonistas y tener percepción de similaridad". }\end{array}$ & $\begin{array}{l}\text { Cognitio- } \\
\text { Afectivo }\end{array}$ & $\begin{array}{l}\text { Estudiantes de } \\
\text { psicología }\end{array}$ \\
\hline 9 & $\begin{array}{c}\text { Empathy Quotient Baron-Cohen } \\
\text { y Wheelwright (2004) }\end{array}$ & Inglaterra & $\begin{array}{l}\text { "La empatía es el impulso o la capacidad de } \\
\text { atribuir estados mentales a otra persona / } \\
\text { animal, y conlleva una respuesta afectiva } \\
\text { apropiada en el observador al estado mental de } \\
\text { la otra persona". }\end{array}$ & $\begin{array}{l}\text { Cognitivo- } \\
\text { Afectivo }\end{array}$ & Adultos \\
\hline 10 & $\begin{array}{c}\text { Basic Empathy Scale } \\
\text { Joliffe y Farrington (2006) }\end{array}$ & Inglaterra & $\begin{array}{l}\text { "Mantener un enfoque en las emociones } \\
\text { básicas, la experiencia de la empatía cognitiva y } \\
\text { afectiva podría ser superior a la de las } \\
\text { emociones no específicas, como estar nervioso, } \\
\text { ansioso o molesto, comúnmente utilizado en } \\
\text { otras escalas". }\end{array}$ & $\begin{array}{l}\text { Cognitivo- } \\
\text { Afectivo }\end{array}$ & Adolescente \\
\hline 11 & $\begin{array}{c}\text { Test of Empathy Scale for } \\
\text { Preeschoolers } \\
\text { Howe, Pit-ten, Brown y Hadwin } \\
\text { (2008) }\end{array}$ & Inglaterra & $\begin{array}{l}\text { "La empatía representa una respuesta cognitiva } \\
\text { y afectiva al estado emocional percibido de otro, } \\
\text { por el cual se propone que un individuo } \\
\text { experimente una reacción emocional similar a } \\
\text { otra persona o a la situación de esa persona" } \\
\text { (Hoffman, 2000; Preston \& de Waal, 2002). }\end{array}$ & $\begin{array}{l}\text { Cognitivo- } \\
\text { Afectivo }\end{array}$ & Preescolares \\
\hline
\end{tabular}

FUENTE: Elaboración propia. 
frontal inferior, la corteza parietal inferior, amígdala, corteza frontal inferior y corteza temporal superior (Figura2) ${ }^{61-63}$

No obstante, por su resolución temporal (milisegundos), el electroencefalograma (EEG) es la técnica utilizada con mayor frecuencia para evaluar el funcionamiento eléctrico cerebra ${ }^{64}$ y su análisis se realiza en los componentes de los potenciales relacionados con eventos (PRE). Gracias a esta técnica, la revisión de la supresión de ondas alfa ha resultado útil en la evaluación de la toma de perspectiva ${ }^{65}$ Por ejemplo, estudios de Perry, Ben-Ami, Lamm, Hoenen, Lubke y Pausse; \& Decety; Héroux, Gray, Joubert, Be Konick, Blanchaette, Beaulieu y De Beaumont; Pelev-Avron, Goldstein, Yellinek, WeismanFogel y Shamay-Toory; y Nishimura, Ikeda y Higuchi ${ }^{66-68}$ han logrado determinar que la supresión de la actividad mu dentro del EEG es un indicador de empatía cognitiva y puede generarse en respuesta a representaciones visuales de otros con dolor e injusticia social.$^{69-71}$ Estas ondas mu (8-12 hz) son un subtipo de ondas alfa que se registran en las áreas motoras del cerebro. Otros estudios que han examinado los rasgos de insensibilidad en la primera infancia, han realizado el análisis del componente de negatividad de ajuste (MMN) el cual es un componente PRE utilizado como índice del grado en que se perciben y procesan las diferencias en la valencia emocional. ${ }^{72}$

De igual modo, la actividad mu ha sido registrada en bebés bajo la hipótesis del desarrollo del funcionamiento del sistema de neuronas espejo vinculadas a esta actividad neuronal y procesos como la comprensión de la acción y la empatía.

Figura 1. Regiones asociadas a la respuesta empática

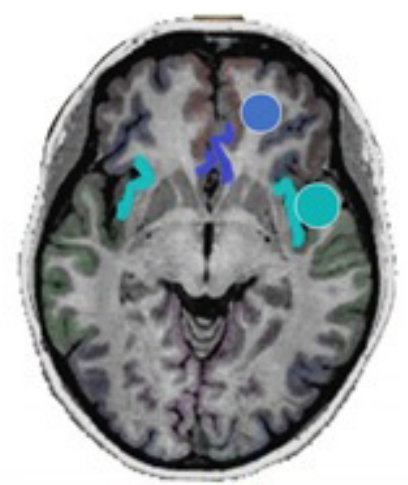

Insula bilateral anterior: Boninick, et a d. (2005) Wing, et al, (2014)

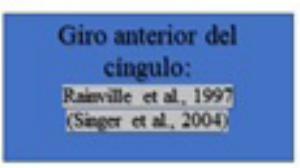

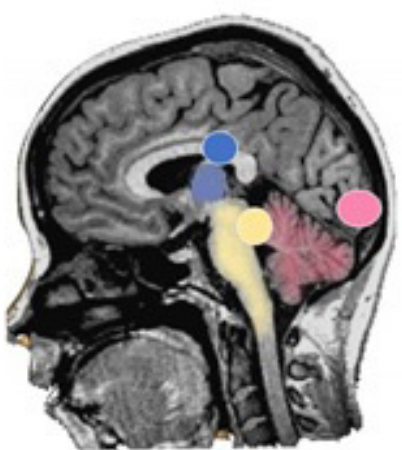

Tronco encefálico: (Singer et al, 2004)
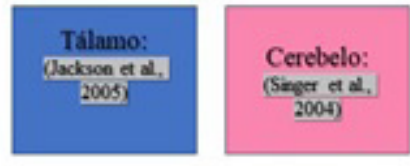

Figura 2. Regiones ligadas al sistema de neuronas espejo observados desde un corte transversal
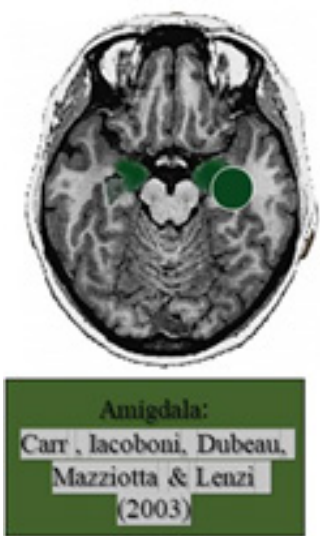
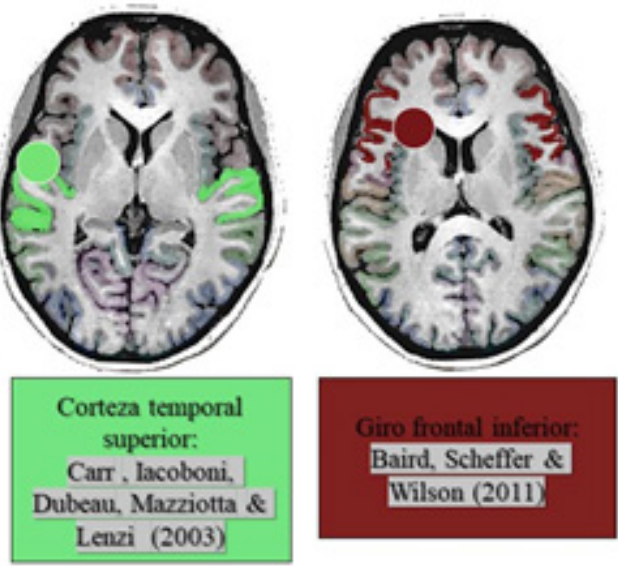

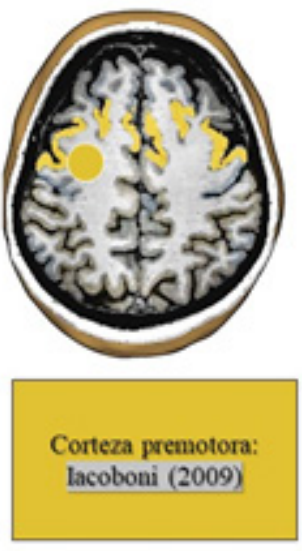


Ejemplo de ello es el trabajo de Nystrom ${ }^{73}$ empleando EEG de alta densidad en 19 lactantes y 15 adultos usando estímulos de video con el agarre en la condición de objetivo dirigido, diseñados para la evitación del reconocimiento facial de emociones en un sujeto que se ubicaba en diferentes distancias de un objeto predeterminado. Oberman y colaboradores ${ }^{74}$ por su parte, realizaron mediciones de la actividad mu y las disfunciones de las neuronas espejo con EEG en una muestra de niños con trastorno del espectro autista utilizando videos de manos y pelotas en movimiento para la imitación de la acción. Ambos procedimientos coinciden en la metodología empleada para el registro de EEG, y los resultados permitieron establecer que existe activación neuronal del mecanismo de neuronas espejo en el caso de los lactantes en la descronización en respuesta al movimiento visual y que el curso temporal de PRE es una herramienta que contribuye a trazar la maduración del sistema de neuronas espejo en los seres humanos desde edad temprana. Por otro lado, la falta de supresión de la onda mu en niños con trastorno del espectro autista sugiere posibles disfunciones en el sistema de neuronas espejo, que implica habilidades como la imitación y la empatía.

Además, las oscilaciones del ritmo mu han sido estudiadas en el procesamiento empático en respuesta a la victimización social, específicamente los prejuicios relacionados con la raza en escenarios de injusticias sociales hacia niños blancos y negros en Estados Unidos. Los resultados muestran que las injusticias sociales como ser objeto de burlas evocan la supresión mu en niños en un rango de edad 5,34 a 8,56 años. Curiosamente no hubo diferencias en la supresión de mu en respuesta a la victimización social en función del grupo racial de la víctima. ${ }^{69}$ Por otro lado, un estudio midió el nivel de moral y mostró que los niños con un índice de evaluación moral más alto demostraron una amplitud de ritmo alfa en general más alta, así como un patrón especifico de amplitud de ritmo theta. Los índices de evaluación moral se correlacionaron con la asimetría alfa en los electrodos F7 y F8 correspondientes prefrontal dorsolateral, lo que indica una mayor activación en las regiones prefrontales del hemisferio izquierdo en niños con una comprensión más desarrollada de la conducta moral. ${ }^{75}$

Avanzando en esta línea, la investigación en bebés lactantes también ha evaluado los correlatos neuronales entre la activación cerebral asimétrica frontal-temporal y el comportamiento prosocial y de consuelo mediante EEG ${ }^{76}$ Las correlaciones plantean una relación entre la activación frontal y las reacciones empáticas de los bebés, al igual que una activación temporal vinculada al comportamiento de ayuda. En contraste con los estudios citados anteriormente, la activación temporal observada no se liga a la desincronización de mu, los reflejos de las acciones del sistema motor o a la habilidad de las personas para com- prender las acciones de su alrededor, teniendo en cuenta que este tipo de activación se centra en áreas centrales sensomotoras del cerebro. Por consiguiente, se resalta la necesidad de comprender la correlación negativa entre la empatía cognitiva y el comportamiento de ayuda, que en otras palabras indica que el comportamiento de ayuda se refleja la orientación a la tarea, mientras que la comprensión de la angustia está relacionada con la orientación social enlazada al análisis del problema del otro.

La técnica de EEG en toma de perspectiva, preocupación empática y comportamiento prosocial en niños preescolares ha demostrado que los análisis de ventanas de N2 producen efectos significativos en los estímulos dolorosos con más amplitudes negativas que neutrales. ${ }^{77}$ Además, los potenciales positivos tempranos y tardíos (LPP) que se han asociado positivamente a comportamiento prosocial de generosidad, evidenciaron efectos del dolor en la LPP temprana, donde las imágenes dolorosas fueron más negativas que neutrales. Los LPP tardíos, en cambio, presentaron interacción de dolor por lateralidad, pero no fueron suficientemente significativos en las pruebas de post-hoc.

La importancia de abordar los estudios de la empatía y sus correlatos con los PRE parte de los aspectos de la primera infancia que se consideran críticos para el desarrollo biológico, psicológico y social del individuo; además de los factores ambientales y familiares que poseen un rol determinantes en la formación y el funcionamiento de estructuras cerebrales. Por lo tanto, la ocurrencia de eventos estresantes y continuos podrían generar en el niño serias dificultades en su desarrollo biopsicosocial. ${ }^{78}$

\section{Conclusión}

La literatura revisada da cuenta de los avances en la medición y conceptualización de la empatía desde su origen hasta la fecha. La creación de instrumentos de forma simultánea con la delimitación del término ha acortado brechas teóricas y metodológicas para ofrecer claridad en el abordaje de este constructo. Los avances en los procesos de desarrollo teórico de la empatía, aunque graduales, han proporcionado un horizonte prometedor para seguir indagando aspectos concernientes a componentes propios de la interacción social en distintos grupos etarios.

Aunque el progreso investigativo de la empatía es notorio, se encontraron pros y contras referentes a las metodologías manejadas en la delimitación de este componente en la población infantil. Conforme a los instrumentos dirigidos a los grupos etarios, es visible la insuficiencia de medidas de empatía para población infantil. Si bien la búsqueda arrojó que se registran dos pruebas elaborados originalmente para niños en edad preescolar y escolar, este resultado involucra afectaciones en la práctica investigativa y clínica al limitar la evaluación de otros dominios relevantes en el transcurso del neurodesarrollo 
de la empatía en la infancia. A pesar de ello, esta situación propone una oportunidad para el avance de nuevos métodos y medidas de las propiedades de la empatía, particularmente en países de habla hispana que comprenden condiciones culturales y sociales que adquieren notable importancia en la cognición para su respectivo estudio.

En la misma línea, se encontró que las pruebas existentes en la recolección de información de la empatía en niños no disponen de contenidos que permitan monitorear el desarrollo de la empatía en el curso de la infancia. La fuerza de la evidencia apunta a que las medidas psicofisiológicas como el EEG tienen la capacidad y la eficacia para este vacío en el conocimiento. Construir herramientas para la vigilancia del progreso de las dimensiones de la empatía junto a instrumentos de recogida de información en la niñez integran un reto especial para el área de la neuropsicología y la evaluación neuropsicológica a nivel ecológico con el objetivo de precisar resultados psicométricos y neurofuncionales según las características individuales del sujeto. Adicionalmente, incorporar instrumentos de medición cognitiva de la empatía a la evaluación psicofisiológica implica evitar desaciertos en la identificación de alteraciones en dicho componente.

Finalmente, este artículo sugiere que el trabajo futuro en población infantil se decante en variables que envuelvan procesos conectados a factores sociales y demográficos como la vulnerabilidad y la inequidad, con el propósito de aportar a nuevos conocimientos que permitan comprender los cambios y dinamismos de la sociedad, debido a que probablemente estos aspectos moldeen las dinámicas de la interacción social y el desenvolvimiento del individuo en la sociedad.

\section{Referencias}

1. Wispé L. History of the concept of empathy. In: Eisenberg N, Strayer J, editors. Empathy and its development. Cambridge University Press; 1987. p. 17-36. DOI: https://doi.org/10.1192/S0007125000224951

2. Decety J, Bartal IBA, Uzefovsky F, Knafo-Noam A. Empathy as a driver of prosocial behaviour: Highly conserved neurobehavioural mechanisms across species. Philos Trans R Soc B Biol Sci. 2016;371(1686). https://doi.org/10.1098/rstb.2015.0077

3. Nook EC, Ong DC, Morelli SA, Mitchell JP, Zaki J. Prosocial Conformity: Prosocial Norms Generalize Across Behavior and Empathy. Personal Soc Psychol Bull. 2016;42(8):1045-62. https://doi. org/10.1177/0146167216649932.

4. Flournoy JC, Pfeifer JH, Moore WE, Tackman AM, Masten CL, Mazziotta JC, et al. Neural Reactivity to Emotional Faces May Mediate the Relationship Between Childhood Empathy and Adolescent Prosocial Behavior. Child Dev. 2016;87(6):1691-702. https://doi.org/10.1111/cdev.12630.
5. Van der Graaff J, Carlo G, Crocetti E, Koot HM, Branje S. Prosocial Behavior in Adolescence: Gender Differences in Development and Links with Empathy. J Youth Adolesc [Internet]. 2018;47(5):1086-99. Available from: http://dx.doi. org/10.1007/s10964-017-0786-1

6. Cosper P, Kaplow R, Moss J. The Impact of Patient and Family Advisors on Critical Care Nurses' Empathy. J Nurs Adm. 2018;48(12):622-8. doi: 10.1097/NNA.0000000000000692.

7. Shao YN, Sun HM, Huang JW, Li ML, Huang RR, Li N. Simulation-Based Empathy Training Improves the Communication Skills of Neonatal Nurses. Clin Simul Nurs. 2018;22:32-42. https://doi.org/10.1016/j. ecns.2018.07.003.

8. Giménez-Espert M del C, Prado-Gascó VJ. The role of empathy and emotional intelligence in nurses' communication attitudes using regression models and fuzzy-set qualitative comparative analysis models. J Clin Nurs. 2018;27(13-14):2661-72. https://doi.org/10.1111/jocn.14325.

9. Denardo Roney JL, Falkenbach DM, Aveson O. Psychopathy and Victim Selection: Does Nonverbal Decoding or Empathy Impact Vulnerability Ratings? J Interpers Violence. 2018;1-22. https://doi. org/10.1177/0886260517742914 .

10. Preston OC, Anestis JC. Psychopathic traits and politics: Examining affiliation, support of political issues, and the role of empathy. Pers Individ Dif [Internet]. 2018;131(April):142-8. Available from: https://doi.org/10.1016/j.paid.2018.04.034

11. Preston SD, De Waal FBM. Empathy: Its ultimate and proximate bases Stephanie D . Preston \& Frans B . M . de Waal Short abstract: Long abstract: Keywords: Table of Contents. Behav brain Sci. 2002;25(1):1-20. https://doi.org/10.1017/ S0140525X02000018.

12. Batson CD. What's wrong with morality? Emot Rev. 2011;3(3):230-6. https://doi. org/10.1177/1754073911402380

13. López MB, Arán V, Richaud M. Empatía: desde la percepción automática hasta los procesos controlados. Av en Psicol Latinoam. 2005;32(1):37-51. Doi: dx.doi.org/10.12804/apl32.1.2014.03 .

14. Dymond BYRF. A scale for the measurement of empathic ability. 1948;127-33. https://doi. org/10.1037/h0061728.

15. Hogan R. Development of an empathy scale. J Consulling Clin Psychol. 1969;33(3):307-16. https://doi. org $/ 10.1037 / \mathrm{h} 0027580$.

16. Block J. The Q-Sort Method in Personality Assessment and Psychiatric Research. 1961. https:// doi.org/10.1037/13141-000.

17. Stotland E. Exploratory Investigations of Empathy. Adv Exp Soc Psychol. 1969;4(C):271-314. https://doi. org/10.1016/S0065-2601(08)60080-5 . 
18. Mehrabian A, Epstein N, Angles L. A measure of emotional empathy '. 1969; https://doi. org/10.1111/j.1467-6494.1972.tb00078.x

19. Davis MH. A multidimensional aprroach to individual differences in empathy. 1980;

20. Baron-cohen S, Wheelwright S. The Empathy Quotient: An Investigation of Adults with Asperger Syndrome or High Functioning Autism , and Normal Sex Differences. 2004;34(2). https://doi.org/10.1023/ B:JADD.0000022607.19833.00.

21. Decety J, Jackson PL. The functional architecture of human empathy. Behav Cogn Neurosci Rev. 2004;3(2):71-100. https://doi. org/10.1177/1534582304267187.

22. Kerr WA, Speroff BJ. Validation and evaluation of the empathy test. J Gen Psychol. 1954;50(2):269-76. https://doi.org/10.1080/00221309.1954.9710125.

23. Bryant BK, Bryant BK. An Index of Empathy for Children and Adolescents. 2014;53(2):413-25. DOI: 10.2307/1128984.

24. Caruso DR, Mayer JD, Caruso DR, Mayer JD(. A Measure of Emotional Empathy for Adolescents and Adults. 1998; ID: 149596869

25. Jolliffe D, Farrington DP. Development and validation of the Basic Empathy Scale. J Adolesc. 2006;29:589611. https://doi.org/10.1016/j.adolescence.2005.08.010.

26. Davis $\mathrm{MH}$. Measuring individual differences in empathy: Evidence for a multidimensional approach. J Pers Soc Psychol. 1983;44(1):113-26. https://doi. org/10.1037/0022-3514.44.1.113.

27. Etain B, Godin O, Boudebesse C, Aubin V, Azorin JM, Bellivier F, et al. Sleep quality and emotional reactivity cluster in bipolar disorders and impact on functioning. Eur Psychiatry. 2017;45:190-7. https:// doi.org/10.1016/j.eurpsy.2017.06.013.

28. Nieri LP. Relación entre la sensibilidad paterna y los estilos de apego, la personalidad y la capacidad empática, según variables sociodemográficas. Psicol Desde El Caribe. 2017;34(1):1-15. http://dx.doi.org/10.14482/ psdc.33.2.7290

29. Lucas-Thompson RG, Holman EA. Environmental stress, oxytocin receptor gene (OXTR) polymorphism, and mental health following collective stress. Horm Behav. 2013;63(4):615-24. https://doi. org/10.1016/j.yhbeh.2013.02.015.

30. Economou M, Peppou LE, Kontoangelos K, Palli A, Tsaliagkou I, Legaki EM, et al. Mental Health Professionals' Attitudes to Severe Mental Illness and Its Correlates in Psychiatric Hospitals of Attica: The Role of Workers' Empathy. Community Ment Health J. 2020;56(4):614-25. https://doi.org/10.1007/s10597019-00521-6.

31. Wang W, Zhou Y, Wang J, Xu H, Wei S, Wang D, et al. Prevalence, clinical correlates of suicide attempt and its relationship with empathy in patients with schizophrenia. Prog Neuro-Psychopharmacology Biol
Psychiatry. 2020;99:109863. https://doi.org/10.1016/j. pnpbp.2020.109863.

32. Jensen K, Gollub RL, Kong J, Lamm C, Kaptchuk TJ, Petrovic P. Reward and empathy in the treating clinician: the neural correlates of successful doctorpatient interactions. Transl Psychiatry. 2020;10(1). https://doi.org/10.1038/s41398-020-0712-2.

33. Fung SC, Zhou S. An Investigation of Pre-Service Teachers' Attitudes Toward Animals and Empathy Toward Humans: Implications for Humane Education Development. Anthrozoos. 2020;33(3):415-26. https://doi.org/10.1080/08927936.2020.1746531.

34. Morganti F, Rezzonico R, Chieh Cheng S, Price CJ. Italian Version of the Scale of Body Connection: Validation and Correlations with the Interpersonal Reactivity Index. Complement Ther Med. 2020;51(March). https://doi.org/10.1016/j.ctim.2020.102400.

35. Ramos-Díaz E, Jiménez-Jiménez V, RodríguezFernández A, Fernández-Zabala A, Axpe I. Emotional intelligence training in a case of an adolescent victim of child maltreatment. con Niños y Adolesc. 2017;4(Figura 1):17-24 ISSN 2340-8340.

36. Carrasco Ortiz MA, Delgado Egido B, Barbero García MI, Holgado Tello FP, del Barrio Gándara MV. [Psychometric properties of the Interpersonal Reactivity Index in Spanish child and adolescent population]. Psicothema. 2011;23(4):824-31. PMID: 22047879.

37. Christopher BT, Farrah GN, Rivera-Hudson N, Paul FJ. Evidence-based assessment of conduct disorder: Current considerations and preparation for DSM-5. Prof Psychol Res Pract. 2013;44(1):56-63. https://doi. org/10.1037/a0029202.

38. Smith S, Dutcher K, Askar M, Talwar V, Bosacki $\mathrm{S}$. Emotional competencies in emerging adolescence: relations between teacher ratings and student self-reports. Int J Adolesc Youth. 2019;24(1):19-28. https://doi.org/10.1080/02673843.2018.1455059.

39. Maoz H, Gvirts HZ, Sheffer M, Bloch Y. Theory of Mind and Empathy in Children With ADHD. J Atten Disord. 2019;23(11):1331-8 https://doi. org/10.1177/1087054717710766.

40. Maoz H, Tsviban L, Gvirts HZ, Shamay-Tsoory SG, Levkovitz Y, Watemberg $\mathrm{N}$, et al. Stimulants improve theory of mind in children with attention deficit/hyperactivity disorder. J Psychopharmacol. 2014;28(3):212-9 https://doi. org/10.1177/0269881113492030.

41. Wang M, Wang J, Deng X, Chen W. Why are empathic children more liked by peers? The mediating roles of prosocial and aggressive behaviors. Pers Individ Dif. 2019;144(February):19-23 https://doi.org/10.1016/j. paid.2019.02.029.

42. Mestre Escrivá V, Frías Navarro MD, Samper García P. La medida de la empatía: análisis del Interpersonal Reactivity Index. Psicothema. 2004;16(2):255-60 ISSN EDICIÓN EN PAPEL: 0214-9915. 
43. Richaud de Minzi MC. Evaluación de la empatía en población infantil argentina. Rev Investig en Psicol. 2008;11(1):101 .DOI: https://doi.org/10.15381/rinvp. v11i1.3880.

44. Barnett MA, Howard JA, King L, Dino GA. Helping behavior and the transfer of empathy. J Soc Psychol. 1981;115:125-32 https://doi.org/10.1080/00224545.19 81.9711995 .

45. Eisenberg-berg N, Mussen P. Empathy and Moral Development in Adolescence. Dev Psychol. 1978;14(2):185-6. https://doi.org/10.1037/00121649.14.2.185

46. Marshall WL, Maric A. Cognitive and emotional components of generalized empathy deficits in child molesters. J Child Sex Abus. 1996;5(2):101-10 https://doi.org/10.1300/J070v05n02_06.

47. Schwenck C, Schmitt D, Sievers S, Romanos M, Warnke A, Schneider W. Kognitive und emotionale Empathie bei Kindern und Jugendlichen mit ADHS und Störung des Sozialverhaltens. Z Kinder Jugendpsychiatr Psychother. 2011;39(4):265-76 https://doi. org/10.1024/1422-4917/a000118.

48. Gorostiaga A, Balluerka N, Soroa G. Evaluación de la empatía en el ámbito educativo y su relación con la inteligencia emocional. Rev Educ. 2012;(364):12-38 DOI: 10.4438/1988-592X-RE-2014-364-253.

49. Sprinkle JE. Animals, empathy, and violence: Can animals be used to convey principles of prosocial behavior to children? Youth Violence Juv Justice. 2008;6(1):47-58. https://doi. org $/ 10.1177 / 1541204007305525$.

50. Howe A, Pit-ten Cate IM, Brown A, Hadwin JA. Empathy in Preschool Children: The Development of the Southampton Test of Empathy for Preschoolers (STEP). Psychol Assess. 2008;20(3):305-9 https://doi.org/10.1037/a0012763.

51. Jackson PL, Meltzoff AN, Decety J. How do we perceive the pain of others? A window into the neural processes involved in empathy. Neuroimage. 2005;24(3):771-9. https://doi.org/10.1016/j.neuroimage.2004.09.006.

52. Singer T, Seymour B, O’Doherty J, Kaube H, Dolan RJ, Frith CD. Empathy for Pain Involves the Affective but not Sensory Components of Pain. Science (80- ). 2004;303(5661):1157-62. DOI: 10.1126/ science.1093535.

53. Botvinick M, Jha AP, Bylsma LM, Fabian SA, Solomon PE, Prkachin KM. Viewing facial expressions of pain engages cortical areas involved in the direct experience of pain. Neuroimage. 2005;25(1):312-9 https://doi.org/10.1016/j.neuroimage.2004.11.043.

54. Lieberman MD. Social cognitive neuroscience: A review of core processes. Annu Rev Psychol. 2007;58:259-89 https://doi.org/10.1146/annurev. psych.58.110405.085654.
55. Wicker B, Keysers C, Plailly J, Royet JP, Gallese V, Rizzolatti G. Both of us disgusted in My insula: The common neural basis of seeing and feeling disgust. Neuron. 2003;40(3):655-64. https://doi.org/10.1016/ S0896-6273(03)00679-2.

56. Preston SD, Bechara A, Damasio H, Grabowski TJ, Stansfield RB, Mehta S, et al. The neural substrates of cognitive empathy. Soc Neurosci. 2007;2(3-4):25475. https://doi.org/10.1080/17470910701376902

57. Xu X, Zuo X, Wang X, Han S. Do you feel my pain? Racial group membership modulates empathic neural responses. J Neurosci. 2009;29(26):8525-9. DOI: https://doi.org/10.1523/JNEUROSCI.2418-09.2009.

58. Wang Q, Zhang Z, Dong F, Chen L, Zheng L, Guo X, et al. Anterior insula GABA levels correlate with emotional aspects of empathy: A proton magnetic resonance spectroscopy study. PLoS One. 2014;9(11):1-11 https://doi.org/10.1371/journal. pone. 0113845 .

59. Rainville P, Duncan GH, Price DD, Carrier B, Bushnell MC. Pain affect encoded in human anterior cingulate but not somatosensory cortex. Science (80). 1997;277(5328):968-71. DOI: 10.1126/ science.277.5328.968.

60. di Pellegrino G, Fadiga L, Fogassi L, Gallese V, Rizzolatti G. Understanding motor events: a neurophysiological study. Exp Brain Res. 1992;91(1):176-80. DOI https://doi.org/10.1007/BF00230027.

61. Baird AD, Scheffer IE, Wilson SJ. Mirror neuron system involvement in empathy: A critical look at the evidence. Soc Neurosci. 2011;6(4):327-35 https:// doi.org/10.1080/17470919.2010.547085.

62. Iacoboni M. Imitation, empathy, and mirror neurons. Annu Rev Psychol. 2009;60:653-70. https:// doi.org/10.1146/annurev.psych.60.110707.163604.

63. Carr L, Iacoboni M, Dubeau M, Mazziotta JC, Lenzi GL. Neural mechanisms of empathy in humans: A relay from neural systems for imitation to limbic areas. Proc Natl Acad Sci. 2003;100(9). https://doi. org/10.1073/pnas.0935845100.

64. Reyes-Haro L, Recasen-Linares A, López-Jiménez $\mathrm{M}$, Fernández-Concepción $\mathrm{O}$, Velez-Siche N, BéjarPazmiño MJ, et al. Uso del Electroencefalograma en un Hospital de Tercer Nivel. Rev Ecuatoriana Neurol. 2016;25(1-3):39-43.

65. Joyal CC, Neveu S-M, Boukhalfi T, Jackson PL, Renaud P. Suppression of Sensorimotor Alpha Power Associated With Pain Expressed by an Avatar: A Preliminary EEG Study. Front Hum Neurosci. 2018;12(July):1-7 https://doi.org/10.3389/ fnhum.2018.00273.

66. Perry A, Bentin S, Bartal IBA, Lamm C, Decety J. "Feeling" the pain of those who are different from us: Modulation of EEG in the mu/alpha range. Cogn Affect Behav Neurosci. 2010;10(4):493-504. DOI https://doi.org/10.3758/CABN.10.4.493. 
67. Suzuki Y, Galli L, Ikeda A, Itakura S, Kitazaki M, Hoyniak CP, et al. Social modeling of eating mediated by mirror neuron activity: A causal model moderated by frontal asymmetry and BMI. Neuropsychologia. 2018;116(1):66-75. https://doi. org/10.1016/j.bbr.2017.10.009.

68. Peled-Avron L, Goldstein P, Yellinek S, WeissmanFogel I, Shamay-Tsoory SG. Empathy during consoling touch is modulated by mu-rhythm: An EEG study. Neuropsychologia. 2018;116(April):68-74 https://doi. org/10.1016/j.neuropsychologia.2017.04.026.

69. Fraser AM, Hampton RS, Spinrad TL, Varnum M, Blais C, Eisenberg N, et al. Children's mu suppression is sensitive to witnessing others' social victimization. Soc Neurosci [Internet]. 2020;00(00):1-7. Available from: https://doi.org/10.1080/17470919.20 20.1722220

70. Cheng Y, Chen C, Decety J. An EEG/ERP investigation of the development of empathy in early and middle childhood. Dev Cogn Neurosci [Internet]. 2014;10:160-9. Available from: http://dx.doi. org/10.1016/j.den.2014.08.012

71. Hoenen M, Lübke KT, Pause BM. Empathic cognitions affected by undetectable social chemosignals: An EEG study on visually evoked empathy for pain in an auditory and chemosensory context. Front Behav Neurosci. 2018;12(October):1-14 https://doi. org/10.3389/fnbeh.2018.00243.

72. Hoyniak CP, Bates JE, Petersen IT, Yang CL, Darcy I, Fontaine NMG. Reduced neural responses to vocal fear: a potential biomarker for callous-uncaring traits in early childhood. Dev Sci. 2018;21(4) https://doi.org/10.1111/desc.12608.
73. Nystrom P. The infant mirror neuron system studied with high density EEG. Soc Neurosci. 2008;3(34):334-47 https://doi.org/10.1080/17470910701563665.

74. Oberman LM, Hubbard EM, McCleery JP, Altschuler EL, Ramachandran VS, Pineda JA. EEG evidence for mirror neuron dysfunction in autism spectrum disorders. Cogn Brain Res. 2005;24(2):190-8 https://doi.org/10.1016/j.cogbrainres.2005.01.014.

75. Orekhova LS, Makhin SA, Mikhailova AA, Pavlenko VB. EEG patterns in early childhood differ between children prone to reward "bad" or "good" actors. Psychol Russ State Art. 2020;13(2):84-95.

76. Paulus M, Kühn-Popp N, Licata M, Sodian B, Meinhardt J. Neural correlates of prosocial behavior in infancy: Different neurophysiological mechanisms support the emergence of helping and comforting. Neuroimage [Internet]. 2013;66:522-30. http:// dx.doi.org/10.1016/j.neuroimage.2012.10.041

77. Decety J, Meidenbauer KL, Cowell JM. The development of cognitive empathy and concern in preschool children: A behavioral neuroscience investigation. Dev Sci. 2018;21(3):1-12. https://doi. org $/ 10.1111 /$ desc. 12570.

78. Bonilla-Santos J, González-Hernández A, BonillaSantos G. Característica Neuroendocrinas del trauma temprano y su relación con el bullying TT - Neuroendocrine characteristics of early trauma and its relation to bullying. Rev Ecuatoriana Neurol [Internet]. 2017;26(3):258-65. Available from: http://scielo.senescyt.gob.ec/scielo.php?script=sci arttext\&pid=S2631-25812017000200258\&lang= pt\%0Ahttp://none/pdf/rneuro/v26n3/2631-2581rneuro-26-03-00258.pdf 